\title{
First Nomogram Predicting the Probability of Lymph Node Involvement in Prostate Cancer Patients Undergoing Radioisotope Guided Sentinel Lymph Node Dissection
}

\author{
Alexander Winter ${ }^{b}$ Thomas Kneib $^{a}$ Martin Rohde ${ }^{c}$ Rolf-Peter Henke ${ }^{d}$ \\ Friedhelm Wawroschek ${ }^{b}$ \\ aWorking Group Statistics and Econometrics, Georg-August University Göttingen, Göttingen, and b University \\ Hospital for Urology, Klinikum Oldenburg, School of Medicine and Health Sciences, Carl von Ossietzky University \\ Oldenburg, ' OFFIS - Institute for Information Technology, ${ }^{d}$ Institute of Pathology Oldenburg, Oldenburg, Germany
}

\section{Key Words}

Prostate cancer · Sentinel lymph node dissection .

Lymphadenectomy · Nomogram · Lymph node involvement

\begin{abstract}
Introduction: Existing nomograms predicting lymph node involvement (LNI) in prostate cancer (PCa) are based on conventional lymphadenectomy. The aim of the study was to develop the first nomogram for predicting LNI in PCa patients undergoing sentinel guided pelvic lymph node dissection (SPLND). Materials and Methods: Analysis was performed on 1,296 patients with PCa who underwent radioisotope guided SPLND and retropubic radical prostatectomy (2005-2010). Median prostate specific antigen (PSA): $7.4 \mathrm{ng} /$ ml (IQR 5.3-11.5 ng/ml). Clinical T-categories: T1: 54.8\%, T2: 42.4\%, T3: $2.8 \%$. Biopsy Gleason sums: $\leq 6: 55.1 \%$, 7: 39.5\%, $\geq 8: 5.4 \%$. Multivariate logistic regression models tested the association between all of the above predictors and LNI. Regression-based coefficients were used to develop a nomogram for predicting LNI. Accuracy was quantified using the area under the curve (AUC). Results: The median number of LNs removed was 10 (IQR 7-13). Overall, $17.8 \%$ of patients $(n=231)$ had LNI. The nomogram had a high predic-
\end{abstract}

tive accuracy (AUC of $82 \%$ ). All the variables were statistically significant multivariate predictors of $\mathrm{LNI}(p=0.001)$. Univariate predictive accuracy for PSA, Gleason sum and clinical stage was 69,75 and $69 \%$, respectively. Conclusions: The sentinel nomogram can predict LNI at a sPLND very accurately and, for the first time, aid clinicians and patients in making important decisions on the indication of a sPLND. The high rate of $L N+$ patients underscores the sensitivity of sPLND.

(c) 2015 S. Karger AG, Basel

\section{Introduction}

Pelvic lymph node dissection (PLND) is still the gold standard for lymph node (LN) staging in clinically localized prostate cancer (PCa). The diagnostic accuracy of available imaging procedures is quite inferior to the histological verification of LN metastases. The LN status is a crucial prognostic factor in PCa. Presence and extension of LN involvement (LNI) is associated with an increased risk of systemic dissemination and progression of the disease. A debate is currently underway on the positive therapeutic impact of PLND, especially in patients with minimal LNI $[1,2]$.

\section{KARGER 125}

(c) 2015 S. Karger AG, Base

0042-1138/15/0954-0422\$39.50/0

E-Mail karger@karger.com

www.karger.com/uin
Dr. med. Alexander Winter

University Hospital for Urology

Carl von Ossietzky University Oldenburg, Klinikum Oldenburg

Rahel-Straus-Strasse 10, DE-26133 Oldenburg (Germany)

E-Mail winter.alexander@ klinikum-oldenburg.de 
Numerous nomograms based on preoperative variables have been developed to predict LNI in PCa and to select candidates for PLNDs. The goal is to identify lowrisk LNI cases and hinder additional morbidity from a PLND. Without exception, these decision tools were based on conventional PLND techniques. Many of these algorithms and Partin tables [3] were based on series in which the patients underwent a limited degree of PLND (IPLND). The nomograms now available are based on ePLND [4-6], which means they account for the fact that LNI prevalence is directly related to the number of dissected LNs and extent of the PLND $[7,8]$. However, the rate of complications rises along with the number of LNs removed [9-11].

Similarly, for radioisotope guided sPLNDs, one can demonstrate a high staging accuracy accompanied by even lower morbidity [11-13]. The sentinel approach allows an individualized extension of LN dissection outside the boarders of ePLND too [14]. Presently, different tracers, such as the near-infrared fluorescent dye indocyanine green, are being tested to mark sentinel LNs (SLNs), especially in connection with robotic [15] and laparoscopic [16] radical prostatectomies (RPs). So far, there is no LNI nomogram based on a sPLND.

We hypothesized that preoperative parameters obtained in patients undergoing sPLNDs can accurately predict LNI in sPLND specimens. To test our hypothesis, we used data collected from men who had undergone a sPLND in combination with a radical retropubic prostatectomy (RRP). Multivariable logistic regression was used to calculate the probability of LNI.

\section{Materials and Methods}

\section{Patients}

A total of 1,325 consecutive patients with PCa were identified, who underwent sPLNDs in combination with RRPs carried out by 4 highly experienced surgeons, in a single center between January 2005 and April 2010. We excluded patients with incomplete clinical information for prostate specific antigen (PSA), clinical stage or biopsy Gleason score $(n=4,0.3 \%)$. Furthermore, we also excluded patients who had undergone a transurethral resection or laser therapy of the prostate $(\mathrm{n}=14,1.1 \%)$ and cT4 tumors $(\mathrm{n}=8$, $0.6 \%)$. An additional 3 patients $(0.2 \%)$ were also excluded, since no SLN could be detected by the gamma probe. The final sample comprised 1,296 patients.

The clinical stage was classified per the 2002 Union for International Cancer Control TNM staging system. PSA was measured using standard assays. The primary pretherapeutic PSA value was considered in patients who had undergone hormonal therapy prior to operative treatment $(\mathrm{n}=12,0.9 \%)$. Prostate biopsies were performed at our hospital, other hospitals and medical offices, which were then examined histopathologically by internal and external uropathology experienced pathologists. All patients were informed orally and in writing about a SPLND and RRP, and they signed a consent form.

\section{SPLND Technique}

Using ultrasound guidance, $99 \mathrm{mTechnetium} \mathrm{nanocolloid} \mathrm{was}$ transrectally injected into the prostate $24 \mathrm{~h}$ before the surgery [12]. Three injections were administered per prostate lobe. Activity attained about $100 \mathrm{MBq}$ per lobe and total injection volume was about $1.2 \mathrm{ml}$. A few hours after injection, scintigraphy was carried out. The radioactivity of the LN was intraoperatively measured using 2 different gamma probe systems (C-Trak System, Care Wise, Morgan Hill, Calif., USA; Crystal Probe SG04, Crystal Photonics GmbH, Berlin, Germany). LNs identified as SLNs by the gamma probe were dissected. For surgical reasons, LNs other than SLNs directly adjoining and adhering to SLNs were also removed, if an in situ separation was not possible. Furthermore, if the SLNs are present in the obturator fossa area, the surrounding non-radioactive lymphatic tissue of the fossa was also dissected. However, the lymphatic tissue of the fossa was not resected, if no SLN existed in the fossa area.

\section{Histopathological Examination}

All LNs were initially cut into 3-mm transverse sections, routinely processed and completely embedded in paraffin; sections of thickness 4-5 $\mu \mathrm{m}$ were stained with hematoxylin-eosin. Selected cases of serial sections were analyzed. An immunohistochemical study with a pancytokeratin antibody (AE1/AE3) was carried out to confirm or exclude metastatic spread in rare cases with inconclusive conventional histology.

\section{Measurement and Statistical Methods}

Univariable and multivariable logistic regression models were carried out to test the association between preoperative tumor characteristics and the probability of LNI. The predictor variables were the preoperative PSA level categorized as 4, 4.1-10, 10.1-20 and $>20 \mathrm{ng} / \mathrm{ml}$; clinical T-category as T1, T2 and T3 and biopsy Gleason sum as 5-6, 7 and 8-10.

Regression coefficients were used to develop the nomogram that predicts the probability of LNI at a sPLND. Bootstrapping (9,999 replications) was applied to generate reliable $95 \%$ confidence intervals for the predicted probabilities and for internal validation. Predictive accuracy was quantified using the receiver operator characteristics of the AUC. The performance characteristics were evaluated using a calibration plot of predicted probabilities against observed LNI rates.

Statistical analyses were performed using the generalized linear model function of the open-source statistical software R (R Development Core Team 2008) [17].

\section{Results}

Table 1 lists the summary of patient characteristics. By definition, details of the Gleason score in the surgical specimen could not be given for 12 patients who previously underwent hormonal treatment. The median number of LNs removed was 10 (interquartile range (IQR) 
Table 1. Patient characteristics

\begin{tabular}{|c|c|c|c|}
\hline & $\begin{array}{l}\text { Overall } \\
(\mathrm{n}=1,296)\end{array}$ & $\begin{array}{l}\mathrm{pN} 0 \\
(\mathrm{n}=1,065,82.2 \%)\end{array}$ & $\begin{array}{l}\mathrm{pN} 1 \\
(\mathrm{n}=231,17.8 \%)\end{array}$ \\
\hline Age at surgery, years & $66(61-70)$ & $66(61-70)$ & $67(62-70)$ \\
\hline Total PSA, ng/ml & $7.4(5.3-11.5)$ & $6.9(5.1-10.0)$ & $12.3(7.3-20.6)$ \\
\hline No. of LN removed & $10(7-13)$ & $10(7-13)$ & $11(9-15)$ \\
\hline No. of positive LN & - & - & $2(1-3)$ \\
\hline \multicolumn{4}{|l|}{ T-category } \\
\hline T1c & $710(54.8)$ & $652(61.2)$ & $58(25.1)$ \\
\hline $\mathrm{T} 2 \mathrm{a}$ & $171(13.2)$ & $136(12.8)$ & $35(15.2)$ \\
\hline $\mathrm{T} 2 \mathrm{~b}$ & $160(12.4)$ & $127(11.9)$ & $33(14.3)$ \\
\hline $\mathrm{T} 2 \mathrm{c}$ & $219(16.9)$ & $135(12.7)$ & $84(36.4)$ \\
\hline $\mathrm{T} 3$ & $36(2.8)$ & $15(1.4)$ & $21(9.1)$ \\
\hline \multicolumn{4}{|l|}{ Biopsy Gleason sum } \\
\hline$\leq 6$ & $714(55.1)$ & $670(62.9)$ & $44(19.1)$ \\
\hline 7 & $512(39.5)$ & $369(34.7)$ & $143(61.9)$ \\
\hline$\geq 8$ & $70(5.4)$ & $26(2.4)$ & $44(19.1)$ \\
\hline \multicolumn{4}{|c|}{ Postoperative Gleason sum, $\mathrm{n}=1,284^{*}$} \\
\hline$\leq 6$ & $269(21.0)$ & $267(25.3)$ & $2(0.9)$ \\
\hline 7 & $942(73.4)$ & $772(73.1)$ & $170(74.6)$ \\
\hline$\geq 8$ & $73(5.7)$ & $17(1.6)$ & $56(24.6)$ \\
\hline \multicolumn{4}{|l|}{ Pathologic stage } \\
\hline pT2 & $841(64.8)$ & $813(76.3)$ & $28(12.1)$ \\
\hline pT3a & $231(17.8)$ & $173(16.2)$ & $58(25.1)$ \\
\hline pT3b & $182(14.0)$ & $68(6.4)$ & $114(49.4)$ \\
\hline pT4 & $42(3.2)$ & $11(1.0)$ & $31(13.4)$ \\
\hline
\end{tabular}

Data are given as median (IQR) or number (\%). * Twelve patients who previously underwent hormonal treatment excluded.

PSA = Prostate specific antigen; $\mathrm{LN}=$ lymph nodes; IQR = interquartile range.

7-13), encompassing a median of 6 (IQR 4-8) SLNs. Overall, $17.8 \%$ of patients $(\mathrm{n}=231)$ had LNI. The number of positive LNs ranged from 1 to 15 (median 2; IQR 1-3).

In the multivariate logistic regression analysis, all variables (pretherapeutic PSA, clinical T-category and biopsy Gleason sum) were significantly associated $(\mathrm{p}<0.001)$ with LNI. The multivariate predictive accuracy (AUC) was $82 \%$, under consideration of the 3 predictors. Univariate analysis also showed a significant $(\mathrm{p}<0.001)$ association between each predictor and LNI. In the univariate predictive accuracy analysis, the biopsy Gleason sum was the most accurate predictor of LNI (74.5\%), followed by the clinical T-category (69.3\%) and the preoperative PSA value $(68.9 \%)$. The results of the multivariate and univariate logistic regression analyses are detailed in table 2 .

Figure 1 illustrates the nomogram tool in a graphical form as generated by the multivariate analysis. The probabilities for LNI, predicted by the multivariate regression analysis, ranged from $3 \%$ in low-risk to $88 \%$ in high-risk PCa patients. For example, the probability of LNI is $24 \%$ for patients with a cT1c tumor, a PSA value of $10 \leq 20$ and a Gleason sum of 7.

The calibration plot of predicted probabilities against observed LNI rates showed a high level of consistency between predicted and actual probabilities in low- and intermediate-predicted probability ranges. Variances from the ideal nomogram are shown in the high-predicted probability ranges (fig. 2 ).

\section{Discussion}

There is general consensus that an ePLND performed on PCa patients achieves the highest staging accuracy. For sPLND, a high staging accuracy has been demonstrated too $[12,13,18]$. LNI predictor ePLND-based nomograms provide $\mathrm{PCa}$ patients a crucial basis to decide for or against a PLND [4-6]. Other predictive models are based on series of IPLNDs, and thereby, these models most likely underestimate the risk of a LNI $[3,19]$. It was 
Table 2. Results of multivariate and univariate logistic regression analyses predicting LN invasion based on preoperative PSA, biopsy Gleason sum and clinical T-category

\begin{tabular}{|c|c|c|c|c|c|}
\hline \multirow[t]{2}{*}{ Predictors } & \multicolumn{2}{|c|}{ Multivariate model } & \multicolumn{2}{|c|}{ Univariate model } & \multirow{2}{*}{$\begin{array}{l}\text { Univariate predictive } \\
\text { accuracy }\end{array}$} \\
\hline & OR & $\mathrm{p}$ value & OR & $\mathrm{p}$ value & \\
\hline Preoperative PSA & - & $<0.001$ & - & $<0.001$ & $68.9 \%$ \\
\hline $4-10 \mathrm{ng} / \mathrm{ml} \mathrm{vs.} \leq 4$ & 1.076 & 0.846 & 0.932 & 0.842 & \\
\hline $10-20 \mathrm{ng} / \mathrm{ml}$ vs. $\leq 4$ & 2.884 & 0.006 & 3.396 & 0.001 & \\
\hline$>20 \mathrm{ng} / \mathrm{ml}$ vs. $\leq 4$ & 3.873 & 0.001 & 6.606 & $<0.001$ & \\
\hline Biopsy Gleason sum & - & $<0.001$ & - & $<0.001$ & $74.5 \%$ \\
\hline 7 vs. $\leq 6$ & 2.586 & $<0.001$ & 5.901 & $<0.001$ & \\
\hline$\geq 8$ vs. $\leq 6$ & 5.062 & $<0.001$ & 25.769 & $<0.001$ & \\
\hline Clinical T-category & - & $<0.001$ & - & $<0.001$ & $69.3 \%$ \\
\hline T2 vs. T1c & 3.786 & $<0.001$ & 4.293 & $<0.001$ & \\
\hline T3 vs. T1c & 12.924 & $<0.001$ & 15.738 & $<0.001$ & \\
\hline Predictive accuracy & $82 \%$ & & & & \\
\hline
\end{tabular}

Fig. 1. Nomogram predicting the probability of lymph node involvement (LNI) in patients undergoing sentinel guided pelvic lymphadenectomy based on the preoperative PSA, clinical T-category and biopsy Gleason sum. Instructions: Locate the pretreatment parameters (e.g. PSA, ng/ml) on the respective axis and draw a line straight up to the point axis. Sum the points for each of the predictors and locate the final sum on the total point axis. Draw a line straight down to find the patient's probability of having a LNI.

\begin{tabular}{|c|c|c|c|c|c|c|c|c|c|c|}
\hline \multicolumn{11}{|c|}{ Points } \\
\hline 0 & 5 & 10 & 15 & 20 & 25 & 30 & 35 & 40 & 45 & 50 \\
\hline \multicolumn{11}{|l|}{ PSA } \\
\hline$\leq 4$ & & & & $10-20$ & $>20$ & & & & & \\
\hline \multicolumn{11}{|c|}{ Clinical stage } \\
\hline $1 \mathrm{c}$ & & & & 2 & & 3 & & & & \\
\hline \multicolumn{11}{|c|}{ Gleason sum } \\
\hline$\leq 6$ & & & & & 7 & & & & $\geq 8$ & \\
\hline \multicolumn{11}{|c|}{ Total points } \\
\hline 0 & 10 & 20 & 30 & 40 & 50 & 60 & 70 & 80 & 90 & 100 \\
\hline \multicolumn{11}{|c|}{ Probability of LNI } \\
\hline 0.03 & 0.05 & 0.08 & 0.13 & 0.21 & 0.32 & 0.45 & 0.58 & 0.71 & 0.81 & 0.88 \\
\hline
\end{tabular}

possible to demonstrate that for a sPLND, the LNI rate was higher in a sentinel cohort than was expected from the European Association of Urology (EAU) guideline nomogram [20]. The validation of a corresponding sentinel-based nomogram is still pending. This study presents the first sPLND-based nomogram.

With an AUC of $82 \%$, the sentinel nomogram presents a comparably accurate model for predicting LNI in patients with PCa. In various IPLND- or ePLND-based nomograms that use the same preoperative parameters as those of predictors of a LNI the reliabilities are $76-86 \%$ $[3,4,6,19]$. Despite the extended approach in these studies, the proportion of $\mathrm{LN}+$ patients was significantly lower than in that of the sentinel series (table 3).

There is no consensus on the risk level of a LNI that would be the ideal cutoff for choosing a PLND in patients with PCa. For instance, the National Comprehensive Cancer Network deems a cutoff acceptable if it leads to waiving about $50 \%$ of the PLNDs prior to RP at the expense of proof or removal of LNMs in $12 \%$ of the cas- 
Table 3. The predictive accuracy of various models predicting LN invasion from PSA, clinical T-category and biopsy Gleason sum

\begin{tabular}{lrlll}
\hline Reference & $\mathrm{n}$ & $\begin{array}{l}\text { PLND } \\
\text { technique }\end{array}$ & $\begin{array}{l}\text { Prevalence } \\
\text { of LNI, \% }\end{array}$ & $\begin{array}{l}\text { AUC, } \\
\%\end{array}$ \\
\hline Makarov et al. [3] & 5,730 & lPLND & 5.0 & 82 \\
Cagiannos et al. [19] & 5,510 & lPLND & 3.7 & 76 \\
Briganti et al. [4] & 602 & ePLND & 11.0 & 76 \\
Godoy et al. [6] & 4,176 & ePLND & 5.2 & 86 \\
Winter et al. & 1,296 & sPLND & 17.8 & 82 \\
\hline
\end{tabular}

IPLND = Limited pelvic lymph node dissection; ePLND $=$ extended PLND; sPLND = sentinel guided PLND; LNI = lymph node involvement.

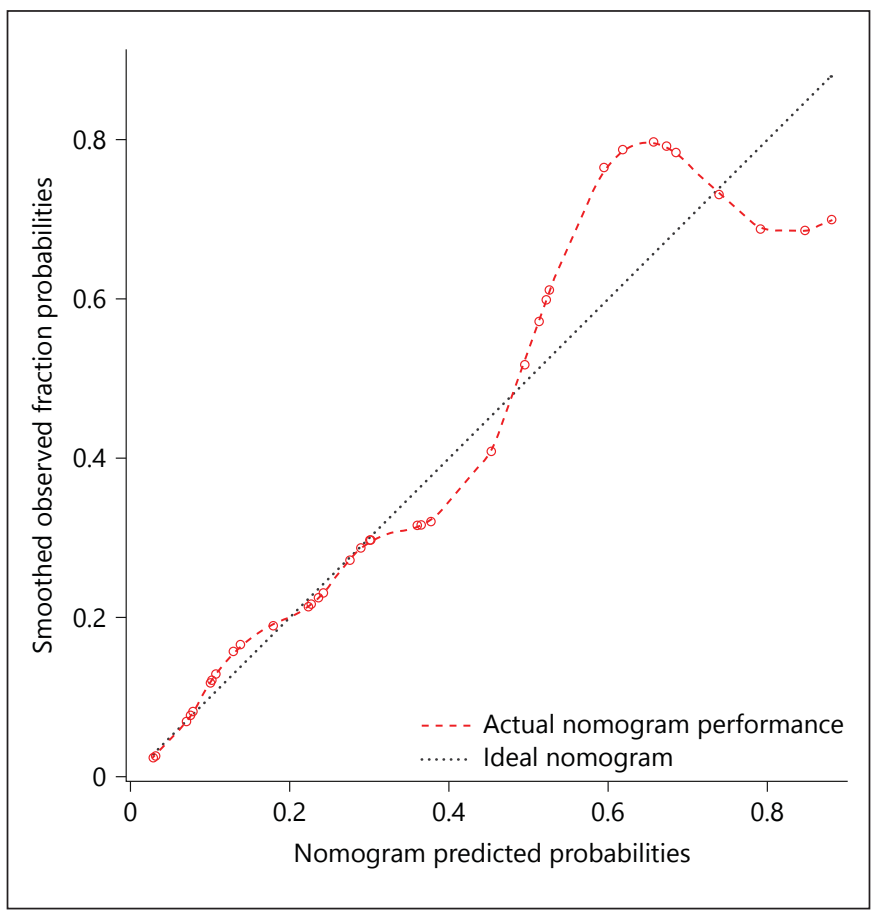

Fig. 2. Nomogram calibration plot. The dotted line indicates the location of the ideal nomogram in which predicted and actual probabilities are identical. The broken line indicates actual nomogram performance.

es with LNI [21]. The EAU guidelines advise the usage of an LNI nomogram-calculated probability of $5 \%$ as a cutoff to perform ePLND, which would allow the avoidance of unnecessary PLND in about $65 \%$ of patients at the cost of missing $12 \%$ of patients with LNI $[5,22]$. Table 4 presents a systematic analysis of a range of nomogram thresholds from 1 to $10 \%$ to help in the correct discrimination of patients with or without histologically confirmed LNI taking into account the sentinel model. The number of avoidable sPLNDs versus the number of potentially missed patients with LNI was quantified. Accordingly, a 7\% threshold would be regarded as the most favorable cutoff. In our population of 1,296 patients, 406 patients $(31.3 \%)$ were classified below this threshold. A voidance of sPLND in those 406 cases would have resulted in missing LNI in 7 patients or in 3\% of all patients with histologically confirmed LNI. Therefore, approximately one-third of patients could be spared from sPLND. Considerable costs and patient discomfort could be saved.

In view of the low morbidity of sPLNDs in combination with the high sensitivity of proof of metastases [11, 13], we question the ability to define a cutoff. One should also note that patients with minimal LNI especially appear to benefit from removal of LN metastases [23]. Finally, the sPLND nomogram offers PCa patients the firstever opportunity to make an informative decision about the probability of the sPLND detecting LN metastases, and thereby allows them to weigh the pros and cons of going for a sPLND for themselves.

On the other hand, a high risk of positive LNs may discourage urologists offering a RP. However, increasing evidence suggests that RP and PLND improve survival in $\mathrm{LN}+\mathrm{PCa}$ [24]. Besides being a staging procedure, PLND may be curative, or at least beneficial, in a subset of patients with limited LNI [23, 25]. A retrospective observational study has shown a dramatic improvement in cancer-specific survival and overall survival in favor of completed RP versus abandoned RP in patients who were found to be LN+ at the time of surgery [26]. These results suggest that RP may have a survival benefit and the discontinuation of RP in $\mathrm{LN}+$ patients may not be justified [22] or that it is useful to perform RP in such cases. Furthermore, RP and PLND are important components of multimodal strategies for patients with $\mathrm{LN}+\mathrm{PCa}$ [22]. Due to these results, we consider the definition of an upper cutoff for PLND as not useful.

No consideration has yet been given to the percentage of positive cores as a predictor, as in other nomograms $[3,5,6,19,27,28]$. In the Update Nomogram of Briganti et al. [5], the percentage of positive cores is the most accurate predictor of LNI. This was also confirmed by external validation studies $[29,30]$. On the flip side, the sentinel nomogram reflects the reality of care. One expects better predictability on inclusion of the percentage of positive cores. Yet, the requirements, such as compliance with standards for biopsies and histopathological preparation, have not yet been established fully in most regions. 
Table 4. Systematic analysis of thresholds used to discriminate between patients with and without histologically confirmed LN involvement in 1,296 patients treated with radical retropubic prostatectomy and radioisotope guided sentinel lymphadenectomy between 2005 and 2010, at a single institution

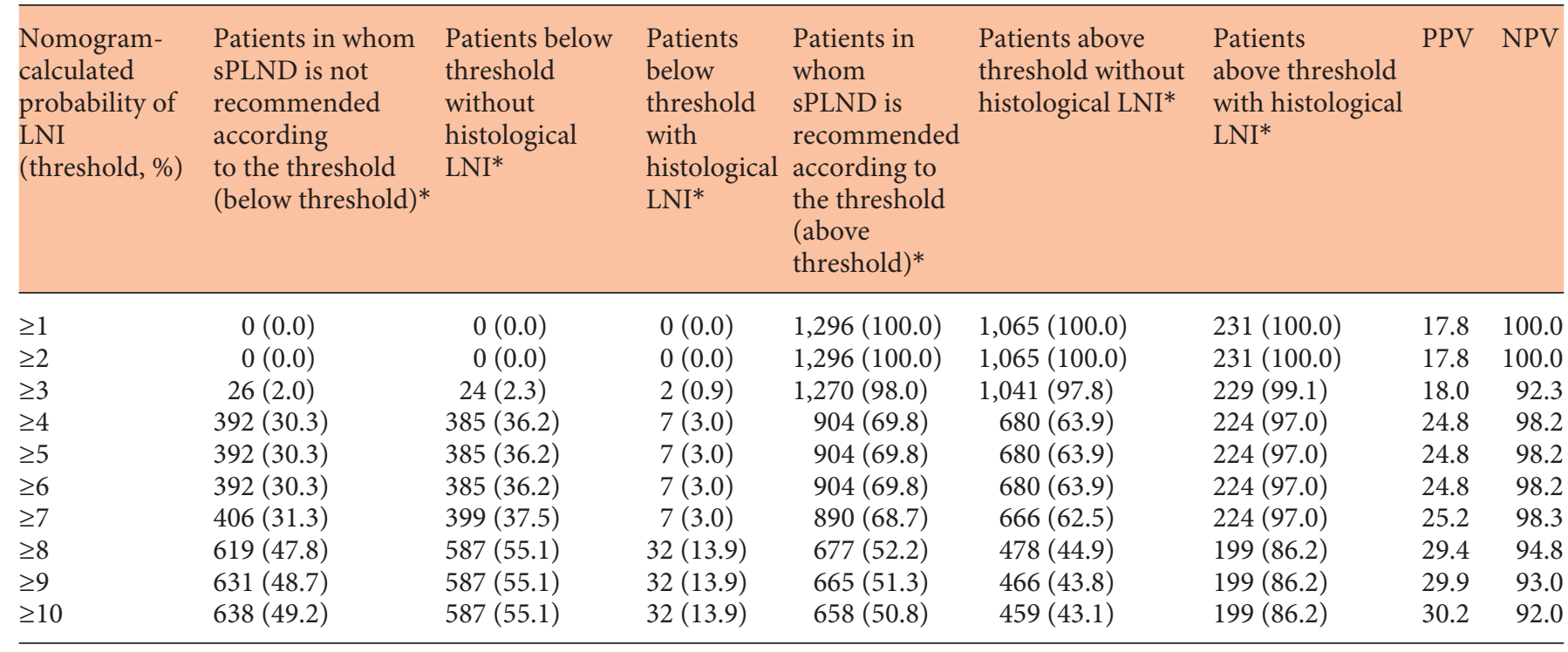

* Data are given as number (\%). PPV = Positive predictive value; NPV = negative predictive value; $\mathrm{LNI}=$ lymph node involvement.

Another limitation of the study arises from the limitations inherent to unicentric analysis. However, the staging accuracy and the rates of LNI patients detected by sPLNDs in the monitored sample compare well with data from other sPLND-experienced centers [13]. Ideally, one should also externally validate the reliability of the sentinel nomogram [29-31].

No clear statement can be made about the sensitivity of a sPLND, since no additional ePLNDs were performed. However, this was not the aim of our research. In a metaanalysis [18], the pooled detection rate of sPLND was $93.8 \%$ with a pooled sensitivity rate of $94 \%$. In the largest study [13] conducted, falsely detected negative results (non-SLNMs found in the absence of SLNMs) were found in $<6 \%$ of the cases.

One fundamental problem with this technique is that when LNs are fully metastasized or when the lymph pathways blocked, the afferent lymph will be directed to other LNs/non-SLNs [32]. These nodes will not be positive on SLN imaging, resulting in false-negative findings. The false-negative rate was shown to correlate with the Gleason score [13]. Patients with a high-risk disease could thus have both positive SLNs and positive non-SLNs [33]. If the goal in such cases is to remove all pelvic LN metastases high-risk patients have the option of undergoing a combination of sPLND and ePLND. As such, the possi-

Sentinel-Based Nomogram Predicting LN Invasion in $\mathrm{PCa}$ bility of an ePLND overlooking a part of the LN metastases, possibly in the pre-sacral region, is overcome by being able to detect it through the sPLND. Reportedly, Joniau et al. [14] did not detect $13 \%$ of metastatic LNs by applying only an ePLND.

\section{Conclusions}

For radioisotope guided sPLNDs, one can demonstrate a high staging accuracy accompanied by even lower morbidity. We have developed the first nomogram to predict the probability of LNI in patients undergoing a sPLND at a RP. The first sentinel nomogram demonstrates a high degree of accuracy. This means that a nomogram can, for the first time, support clinicians and patients in making a key decision on whether to go for a sPLND. Compared with the ePLND-based nomograms, the higher rate of $\mathrm{LN}+$ patients detected underpins the sensitivity of the sPLND. An external validation of the sentinel nomogram is still pending.

\section{Acknowledgements}

None. 


\section{References}

1 Withrow DR, DeGroot JM, Siemens DR, Groome PA: Therapeutic value of lymph node dissection at radical prostatectomy: a population-based case-cohort study. BJU Int 2011;108:209-216.

2 Schumacher MC, Burkhard FC, Thalmann GN, Fleischmann A, Studer UE: Good outcome for patients with few lymph node metastases after radical retropubic prostatectomy. Eur Urol 2008;54:344-352.

3 Makarov DV, Trock BJ, Humphreys EB, et al: Updated nomogram to predict pathologic stage of prostate cancer given prostate-specific antigen level, clinical stage, and biopsy Gleason score (Partin tables) based on cases from 2000 to 2005. Urology 2007;69:10951101.

4 Briganti A, Chun FK, Salonia A, et al: Validation of a nomogram predicting the probability of lymph node invasion among patients undergoing radical prostatectomy and an extended pelvic lymphadenectomy. Eur Urol 2006;49:1019-1026.

5 Briganti A, Larcher A, Abdollah F, et al: Updated nomogram predicting lymph node invasion in patients with prostate cancer undergoing extended pelvic lymph node dissection: the essential importance of percentage of positive cores. Eur Urol 2012;61:480-487.

6 Godoy G, Chong KT, Cronin A, et al: Extent of pelvic lymph node dissection and the impact of standard template dissection on nomogram prediction of lymph node involvement. Eur Urol 2011;60:195-201.

7 Bader P, Burkhard FC, Markwalder R, Studer UE: Is a limited lymph node dissection an adequate staging procedure for prostate cancer? J Urol 2002;168:514-518.

8 Heidenreich A, Ohlmann CH, Polyakov S: Anatomical extent of pelvic lymphadenectomy in patients undergoing radical prostatectomy. Eur Urol 2007;52:29-37.

9 Briganti A, Chun FK, Salonia A, et al: Complications and other surgical outcomes associated with extended pelvic lymphadenectomy in men with localized prostate cancer. Eur Urol 2006;50:1006-1013.

10 Musch M, Klevecka V, Roggenbuck U, Kroepfl D: Complications of pelvic lymphadenectomy in 1,380 patients undergoing radical retropubic prostatectomy between 1993 and 2006. J Urol 2008;179:923-928.

11 Winter A, Vogt C, Weckermann D, Wawroschek F: [Complications of pelvic lymphadenectomy in clinically localised prostate cancer: different techniques in comparison and dependency on the number of removed lymph nodes]. Aktuelle Urol 2011;42:179183.

12 Wawroschek F, Vogt H, Weckermann D, Wagner T, Hamm M, Harzmann R: Radioisotope guided pelvic lymph node dissection for prostate cancer. J Urol 2001;166:1715-1719.

13 Holl G, Dorn R, Wengenmair H, Weckermann D, Sciuk J: Validation of sentinel lymph node dissection in prostate cancer: experience in more than 2,000 patients. Eur J Nucl Med Mol Imaging 2009;36:1377-1382.

14 Joniau S, Van den Bergh L, Lerut E, et al: Mapping of pelvic lymph node metastases in prostate cancer. Eur Urol 2013;63:450-458.

15 Jeschke S, Lusuardi L, Myatt A, Hruby S, Pirich C, Janetschek G: Visualisation of the lymph node pathway in real time by laparoscopic radioisotope- and fluorescence-guided sentinel lymph node dissection in prostate cancer staging. Urology 2012;80:1080-1086.

16 Manny TB, Patel M, Hemal AK: Fluorescence-enhanced robotic radical prostatectomy using real-time lymphangiography and tissue marking with percutaneous injection of unconjugated indocyanine green: the initial clinical experience in 50 patients. Eur Urol 2014;65:1162-1168.

17 R Development Core Team (2011): R: A Language and Environment for Statistical Computing. Vienna, Austria, R Foundation for Statistical Computing. http://www.R-project. org/.

18 Sadeghi R, Tabasi KT, Bazaz SM, et al: Sentinel node mapping in the prostate cancer. Meta-analysis. Nuklearmedizin 2011;50:107115.

19 Cagiannos I, Karakiewicz P, Eastham JA, et al: A preoperative nomogram identifying decreased risk of positive pelvic lymph nodes in patients with prostate cancer. J Urol 2003;170: 1798-1803.

20 Winter A, Kneib T, Henke RP, Wawroschek F: Sentinel lymph node dissection in more than 1200 prostate cancer cases: rate and prediction of lymph node involvement depending on preoperative tumor characteristics. Int J Urol 2014;21:58-63.

21 Mohler J, Bahnson RR, Boston B, et al: NCCN clinical practice guidelines in oncology: prostate cancer. J Natl Compr Canc Netw 2010;8: 162-200.

22 Mottet N, Bastian PJ, Bellmunt J, et al: EAU Guidelines on Prostate Cancer, 2014. http:// www.uroweb.org/gls/pdf/1607\%20Prostate\%20Cancer_LRV3.pdf.

23 Briganti A, Karnes JR, Da Pozzo LF, et al: Two positive nodes represent a significant cut-off value for cancer specific survival in patients with node positive prostate cancer. A new proposal based on a two-institution experience on 703 consecutive $\mathrm{N}+$ patients treated with radical prostatectomy, extended pelvic lymph node dissection and adjuvant therapy. Eur Urol 2009;55:261-270.

24 Gakis G, Boorjian SA, Briganti A, et al: The role of radical prostatectomy and lymph node dissection in lymph node-positive prostate cancer: a systematic review of the literature. Eur Urol 2014;66:191-199.

25 Seiler R, Studer UE, Tschan K, et al: Removal of limited nodal disease in patients undergoing radical prostatectomy: long-term results confirm a chance for cure. J Urol 2014;191: 1280-1285.

26 Engel J, Bastian PJ, Baur H, et al: Survival benefit of radical prostatectomy in lymph nodepositive patients with prostate cancer. Eur Urol 2010;57:754-761.

27 Briganti A, Karakiewicz PI, Chun FK, et al: Percentage of positive biopsy cores can improve the ability to predict lymph node invasion in patients undergoing radical prostatectomy and extended pelvic lymph node dissection. Eur Urol 2007;51:1573-1581.

28 Heidenreich A, Pfister D, Thüer D, Brehmer B: Percentage of positive biopsies predicts lymph node involvement in men with lowrisk prostate cancer undergoing radical prostatectomy and extended pelvic lymphadenectomy. BJU Int 2011;107:220-225.

29 Hinev AI, Anakievski D, Kolev NH, Hadjiev VI: Validation of nomograms predicting lymph node involvement in patients with prostate cancer undergoing extended pelvic lymph node dissection. Urol Int 2014;92:300305.

30 Gacci M, Schiavina R, Lanciotti M, et al: External validation of the updated nomogram predicting lymph node invasion in patients with prostate cancer undergoing extended pelvic lymph node dissection. Urol Int 2013; 90:277-282.

31 Shariat SF, Karakiewicz PI, Suardi N, Kattan MW: Comparison of nomograms with other methods for predicting outcomes in prostate cancer: a critical analysis of the literature. Clin Cancer Res 2008;14:4400-4407.

32 Morgan-Parkes JH: Metastases: mechanisms, pathways, and cascades. AJR Am J Roentgenol 1995;164:1075-1082.

33 Weckermann D, Dorn R, Holl G, Wagner T, Harzmann R: Limitations of radioguided surgery in high-risk prostate cancer. Eur Urol 2007;51:1549-1556. 